\title{
Prevention of Cavitation in Propellers
}

Citation: M. Taseidifar, J. Antony, R.M. Pashley (2020) Prevention of Cavitation in Propellers. Substantia 4(2) Suppl.: 109-117. doi: 10.36253/Substantia-821

Copyright: (c) 2020 M. Taseidifar, J. Antony, R.M. Pashley. This is an open access, peer-reviewed article published by Firenze University Press (http://www.fupress.com/substantia) and distributed under the terms of the Creative Commons Attribution License, which permits unrestricted use, distribution, and reproduction in any medium, provided the original author and source are credited.

Data Availability Statement: All relevant data are within the paper and its Supporting Information files.

Competing Interests: The Author(s) declare(s) no conflict of interest.

\author{
Mojtaba Taseidifar ${ }^{1}$, John Antony ${ }^{2}$, Richard M. Pashley ${ }^{1, *}$ \\ ${ }^{1}$ School of Science, UNSW Canberra, Northcott Drive, Canberra, Australia \\ ${ }^{2}$ Dynamikx Pty Ltd, 169 Hampton Road, South Fremantle, WA, Australia \\ *Corresponding author: r.pashley@adfa.edu.au
}

\begin{abstract}
It is well known that cavitation phenomena affect the efficiency of propellers. It is a major worldwide economic problem for the transport industry. The speed of fast, ocean going vessels is limited by cavitation effects on hydrofoils and propulsion systems. The main approaches by industry to mitigate the detrimental effects of cavitation on propellers is restricted to varying operating conditions, geometric design and employing wear resistant materials. We here develop a simple solution to the problem. It has been known for over a century that dissolved gases reduce the tensile strength of liquids by orders of magnitude. Degassing a liquid dramatically reduces its ability to cavitate. Propeller cavitation in ships and submarines is typically controlled by reducing rotation rate and/or blade pitch. We here demonstrate the astonishing fact that cavitation can be completely prevented by releasing degassed water adjacent to the low pressure side of a rotating propeller, without varying blade speed or pitch. Practical implementation is simple and cheap.
\end{abstract}

Keywords: cavitation, degassing, hollow fibre membrane, propeller.

\section{INTRODUCTION}

At the end of the $19^{\text {th }}$ century it was realised that ships were not attaining their projected design speed. This was eventually found to be due to cavitation. ${ }^{1}$ The effects of the collapse of a spherical cavity within a fluid were first considered by Besant in 1859. ${ }^{2}$ Cavitation in fluids has been studied for over a century since this pioneering work and that of Reynolds in 1886 and Lord Rayleigh in $1917 .^{3}$ There are two main types: inertial cavitation, created by differences between boundary and bulk fluid flow in pumps, valves and propellers; and non-inertial cavitation, created by oscillatory processes such as simple shaking and sonication. ${ }^{1}$ Cavitation also occurs in fine cavities between solid surfaces. ${ }^{4}$ In many diverse processes, cavitation not only reduces the efficiency of fluid systems but the collapse of the created bubbles near surfaces creates shock waves. Shock waves create microjets which impinge upon a surface and create wear. The temperatures produced by the rapid collapse of a bubble can reach 20,000 degrees $\mathrm{K}$, and can cause transient light emission or sonoluminescence, and initiate undesirable reactions in fluid components. The cavitation index $C$ ( $\sigma$ or sometimes $k$ ) is used as a measure of cavitation potential and is defined as: ${ }^{5}$ 
$C \equiv \frac{\left(p_{r}-p_{v}\right)}{\frac{1}{2} \rho V^{2}}$

where $p_{r}-p_{v}$ is the pressure difference due to dynamic effects of the fluid flow; $p_{r}$ is the local (reduced reference pressure) and $p_{v}$ the vapour pressure of the fluid (at that temperature). $\rho$ is the density of the fluid and $V$ the fluid velocity. In effect, the cavitation index is the ratio of the work done by the pressure to the work done by the fluid kinetic energy. The critical value of the index $C_{i}$ is when cavitation inception occurs, and $C<C_{i}$ corresponds to conditions of advanced cavitation. The ability of a particular fluid to cavitate depends on the tensile strength of the fluid under the operating conditions. In general, $C$ > 1 makes cavitation increasingly unlikely. ${ }^{6}$

In fluid cavitation, it is generally assumed that local suction pressures just below the vapour pressure of a fluid, at a given temperature, will nucleate bubbles which then implode once local hydrostatic pressure returns. In practice, there is a significant additional barrier to the formation of cavities in the absence of suitable nucleation sites, and it is actually very difficult to cavitate pure liquids in clean, smooth vessels. Nano-sized cavities are usually the smallest structures which can be considered as a separate phase, and their growth or collapse controls the extent of cavitation. ${ }^{4}$ The presence of dissolved atmospheric gases facilitates fluid cavitation. For example, water can dissolve close to $20 \mathrm{~mL}$ of atmospheric gases per litre and hydrocarbons typically absorb ten times more. The removal of these dissolved gases inhibits fluid cavitation.

In 1982, Israelachvili and $\mathrm{Pashley}^{7}$ made the remarkable discovery that hydrophobic surfaces immersed in water were attracted to each other with a long-range $(\sim 10 \mathrm{~nm})$ force much stronger than expected for van der Waals forces. They called this the 'longrange hydrophobic interaction'. Since that discovery, there have been many reports extending the range of the force, depending on surfaces and conditions, in some cases to a range of $300 \mathrm{~nm}$. These observations created a problem for theoretical interpretation simply because the local effect of a hydrophobic, nonhydrogen bonding surface on water should only extend a few water molecules, at most. In 1985, Pashley et al. ${ }^{4}$ noticed that a bridging cavity was formed when two solid hydrophobic surfaces were pulled apart in water, and later Christenson et al. ${ }^{8}$ reported cavitation as two hydrophobic surfaces approached within a few $\mathrm{nm}$, but before making contact. These observations led to the suggestion that the long-range hydrophobic interaction may be caused by the formation of bridging cavities between hydrophobic surfaces, giving a more reason- able explanation for the extraordinarily long range of the force.

Craig et al. ${ }^{9}$ have given a thorough review of bulk and surface cavitation and its link to nanobubbles. Two recent studies ${ }^{10,11}$ have also considered the formation and applications of bulk nanobubbles.

If cavitation held hydrophobic surfaces together, then it follows that the inhibition of cavitation by removing dissolved atmospheric gases may indeed allow oil and water to mix. This idea was tested by Pashley (in 2003) ${ }^{12}$ by studying the effects of degassing on the dispersion of oil droplets in water. Hydrocarbon oils such as decane immediately phase separated after shaking with water but, upon degassing, a fine stable dispersion was easily produced. This work demonstrated that cavitation also plays a central role in the dispersion of oil in water.

We note in passing that with colloidal systems involving electrolytes (DLVO) forces and Hofmeister effects change dramatically with removal of dissolved gas, a fundamental matter attracting much interest.

The presence of dissolved, non-polar gas molecules in a surrounding fluid, in equilibrium with the atmosphere, produces nucleation sites throughout this fluid. Both aqueous and non-aqueous fluids show similar effects, and, in addition, non-polar fluids have an increased capacity to dissolve non-polar gases relative to water. It has been demonstrated ${ }^{13}$, and is elucidated upon in this work, that degassing a separate quantity of the fluid, and releasing it such that it flows directly onto a moving surface can completely prevent cavitation by providing a boundary layer of degassed fluid on the surface.

\section{MATERIALS AND METHODS}

\subsection{Materials}

Hollow fibre membranes were purchased from Membrana, Charlotte, USA (Model $2 \times 6$ Radial Flow Superphobic). A vacuum pump, Fossa FO 0015 A (Busch Sydney, Australia) was used for de-gassing. A diaphragm water pump (model: FloJet-D3732-E5011) was purchased from CreativePumps Australia. A Mettler-Toledo M700 process analysis system fitted with a type O2 $4700 \mathrm{ppb}$ module to detect dissolved oxygen levels in liquids in ppb, and fitted with a corresponding InPro 6900 dissolved oxygen electrode was obtained from MettlerToledo Ltd., Melbourne, Australia. Tap water with and without added salt $(\mathrm{NaCl}$ with $0.17 \mathrm{M})$ was used in all the experiments. 


\subsection{Methods}

2.2.1. Membrane degassing for water and aqueous solutions

Hollow fibre hydrophobic membrane systems offer the most efficient commercial process for large scale degassing due to providing high surface area of contact per unit volume of fluid. ${ }^{14}$ A photograph of the experimental setup used in this study is given in Figure 1. A strong vacuum is applied to the outside of a hollow fibre hydrophobic membrane or membrane array where the dissolved gases emerge, while the aqueous solution flows around a core of hollow fibres. The hollow fibre membranes are strongly hydrophobic (Teflon or polypropylene), and have small pores designed to prevent liquid water passing through them due to the high Laplace pressure of small diameter water/air interfaces. Only water vapour can exist in the pores and the high surface area membrane efficiently transfers water vapour and atmospheric gases out of aqueous solutions. This technique has been used to produce a continuous flow of water more than $99.5 \%$ degassed.

In these experiments, tap water was used to determine the effect of degassing on cavitation, and gases were removed from the tap water by pumping it through the membrane using a small diaphragm water pump while applying a vacuum to the inside of the hollow fibres in the membrane. The vacuum pump, with ultimate pressure $\leq 2.5 \times 10^{-2}$ mbar was protected from exposure to water vapour by two $5 \mathrm{~L}$ pyrex glass tanks con-

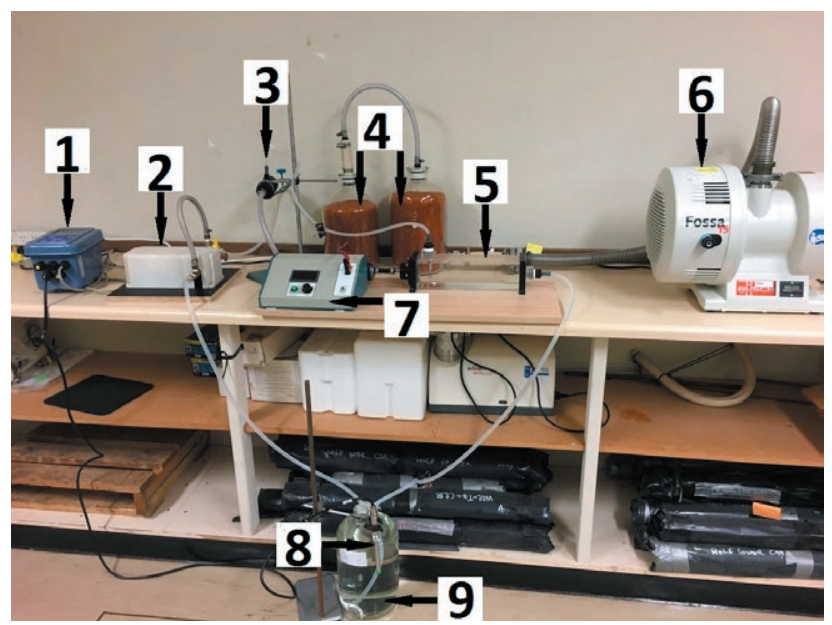

Figure 1. Photograph of the system used to study the effect of degassing on cavitation. (1) M700 process analysis system (to monitor DO levels); (2) Hollow fibre membrane; (3) Water pump; (4) Silica gel tanks; (5) Observation cell; (6) Vacuum pump; (7) Variable motor; (8) DO electrode; (9) Degassed water reservoir. nected in series filled with pre-dried granular silica gel. The dissolved oxygen content, and thereby the level of degassing, was measured using an InPro 6900 Oxygen electrode with a detection limit of lppb in these studies.

Cavitation was monitored visually using a Pentax K-5 II S camera aimed at an observation cell, shown in Figure 1. The observation cell (5) was a perspex housing (L: $300 \mathrm{~mm}, \mathrm{~W}: 65 \mathrm{~mm}$ and $\mathrm{H}: 65 \mathrm{~mm}$ ) for a three-blade propeller $(5 \mathrm{~cm}$ diameter) inside and two metal tubes sealed to the box - one to provide normal gassed water (tap water or salt water) and another one to provide degassed water.

\section{RESULTS AND DISCUSSION}

\subsection{Theoretical prediction of the link between degassing and cavitation pressure}

In fluid cavitation, it is generally assumed that local suction pressures just below the vapour pressure of the fluid, at a given temperature, will nucleate bubbles which then implode once local hydrostatic pressure returns. In practice, there is a significant additional barrier to the formation of cavities in the absence of suitable nucleation sites, and it is actually very difficult to cavitate pure liquids in clean, smooth vessels. Nano-sized cavities are usually the smallest structures which can be considered as a separate phase, and their growth or collapse controls the extent of cavitation. ${ }^{12}$ Both homogeneous cavitation and heterogeneous nucleation cavitation in water are considered here, caused by the presence of inert dissolved atmospheric gas molecules throughout the bulk liquid phase.

The barrier to 'ideal' homogeneous cavitation can be estimated from a simple analysis of the formation of a nano-sized spherical cavity. The total energy $E_{T}$ of a cavity of radius $r$ is given by the sum of the negative work done by the suction pressure $-\Delta P$ on the cavity volume and the surface tension work done on creating the surface of the cavity. Thus, the total cavity energy is given by:

$E_{T}=\frac{4}{3} \pi r^{3}(\Delta P)+4 \pi r^{2} \gamma$

A diagram of the behaviour expected for water is shown in Figure 2. Assuming that $\mathrm{nm}$-sized cavities must form within pure water, the barrier to their formation is very high, of the order of $80 \mathrm{kT}$, which makes their formation difficult.

If we make the assumption that $1 \mathrm{~nm}$ is the critical radius of cavity formation, i.e. when $d E_{T} / d r=0$, we can estimate the critical suction pressure from the Laplace equation: 


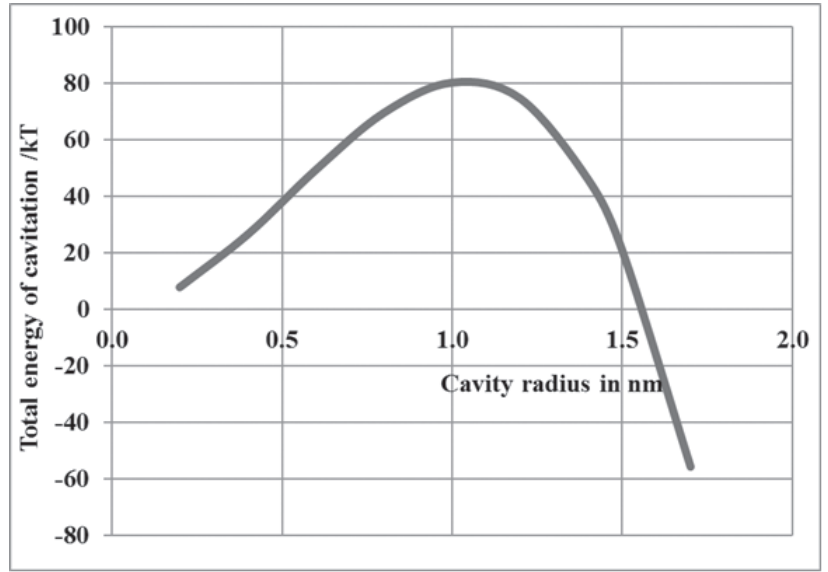

Figure 2. Theoretical calculation of the energy (in $k T$ units) required to form a spherical cavity of radius $r$ in pure water under ideal, de-gassed conditions, in the absence of nucleation sites, with an applied suction pressure of $-1400 \mathrm{~atm}$.

$\Delta P=-\frac{2 \gamma}{r_{c}}$

This gives a critical suction pressure of about $-1,440$ atm for pure water or -493 atm for a typical hydrocarbon liquid. The largest suction pressure observed experimentally for degassed water was -1400 atm, which is close to theoretical predictions. ${ }^{16}$

In most practical situations, contaminants and 'real', rough surfaces facilitate the heterogeneous nucleation of cavities in water at much lower suction pressures than this. The presence of dissolved gases and hydrophobic groups also substantially reduce the cavitation pressure. Experimental cavitation pressures are typically about -1 atm for distilled water, saturated with air, since this is effectively the vapour pressure of water at room temperature. However, a suction pressure of $-200 \mathrm{~atm}$ is required when the water is $99.98 \%$ de gassed according to ${ }^{17}$; see Figure 3. From these experimental results, it is clear that degassing water strongly inhibits cavitation, especially when degassed to greater than $99 \%$. The disruptive presence of dissolved, non-polar gas molecules in the liquid water phase produces nucleation sites throughout the liquid. ${ }^{4}$

From solid state physics it is well known from studies of lattice dynamics in the harmonic approximation isotopes or impurities attract each other, a collective effective van der Waals interaction and associate into aggregates. The same happens in liquids. It is these that act as nucleation sites that connect adjoining nano sites. An equivalent result occurs with non-aqueous fluids, which also have an increased capacity to dissolve non polar gases relative to water.

A theoretical model has been developed to estimate the cavitation pressure $p_{c}$ required to cause (heteroge- neous) cavitation in water at a wide range of dissolved gas levels. The basic principle used is that the pressure required can be estimated from the change in activation energy $\Delta \mu$ required to transfer a dissolved gas molecule (e.g. $\mathrm{N}_{2}$ ) from the aqueous phase to the gas phase. Since $\mu(g, w)$ will change with concentration of the dissolved gas in water $x_{g}$, i.e.:

$\mu(g, w)=\mu^{0}(g, w)+k T \ln x_{g}$

and since the gas-phase chemical potential will stay constant, the activation energy for the transfer from solution to gas phase will vary with concentration in the aqueous solution as:

$\Delta \mu=k \operatorname{Tln}\left(\frac{x_{g}^{S}}{x_{g}}\right)$

where $x_{g}^{s}$ is the mole fraction of gas in water under standard atmospheric conditions (1 atm).

The cavitation pressure can then be estimated from the activation-energy equation:

$p_{c}=p_{0} \exp \left(\frac{\Delta \mu}{k T}\right)$

where $p_{0}$ is the standard, i.e. saturated gas cavitation value, of 1 atm.

Hence, it follows that:

$p_{c}=p_{0}\left(\frac{x_{g}^{s}}{x_{g}}\right)$

Note that for air-equilibrated water, $x_{g}{ }^{s}$ is about 1.53 $\times 10^{-5}$.

Also, note that this result reduces simply to:

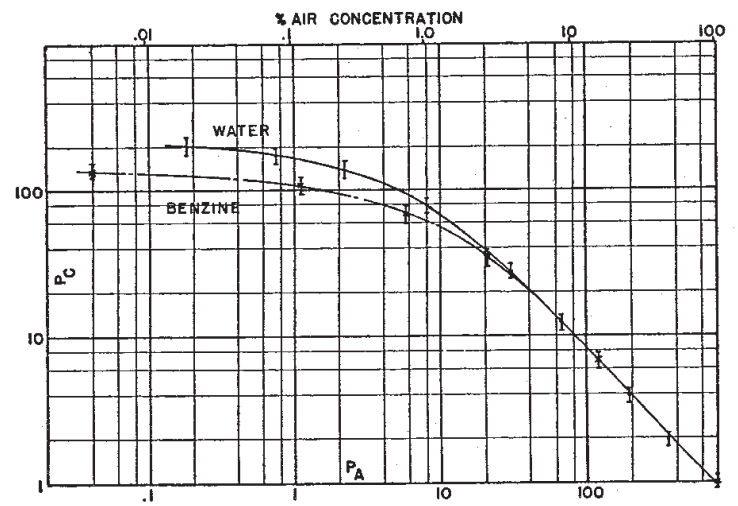

Figure 3. Experimental data showing the effect of de-gassed levels on the suction pressures required to produce cavitation in water and benzene. Reprinted with permission from Ref 16. Copyright 1954, Acoustic Society of America. 
$p_{c}(a t m)=\left(\frac{100}{100-\% d e G}\right)$

This cavitation-pressure model will work for any liquid, since it is based entirely on gas solubility relative to a standard state. A graphical representation of this result is given in Figure 4 and Figure 5.

For the case of water, the model predicts that when the dissolved gas level is reduced to about $0.07 \%$ (or about $0.6 \mu \mathrm{M})$, the gas cavitation pressure equals that of pure water (note that at $20{ }^{\circ} \mathrm{C}$, water in equilibrium with the atmosphere has $0.85 \mathrm{mM}$ of dissolved gases: $\mathrm{N}_{2}, \mathrm{O}_{2}, \mathrm{CO}_{2}$ and $\mathrm{Ar}$ ). The pure water cavitation pressure is at its maximum and can be estimated using the Laplace equation with an estimated, critical cavity radius of $1 \mathrm{~nm}$ (eq. 3).

This gives a calculated (homogeneous) cavitation pressure of -1440 atm. This value agrees with the reported experimental value for pure (i.e. completely degassed) water of $-1400 \mathrm{~atm}$. These high suction pressures are also consistent with the basic (kinetic) model of con-

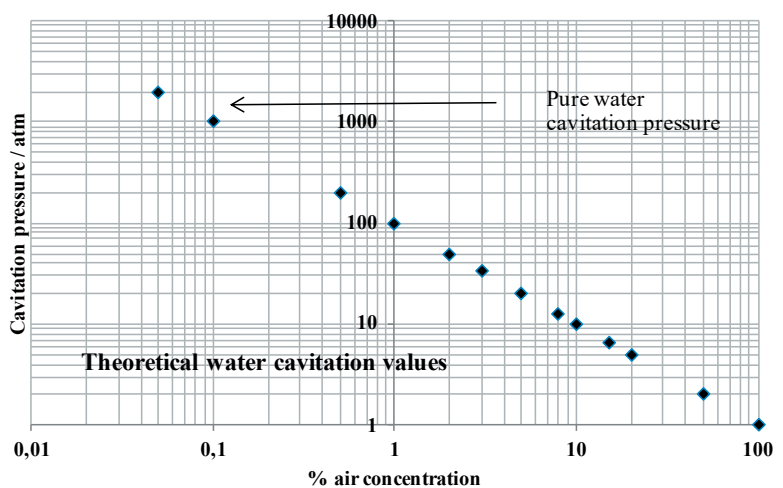

Figure 4. Calculated cavitation pressures for water obtained using Equation (8).

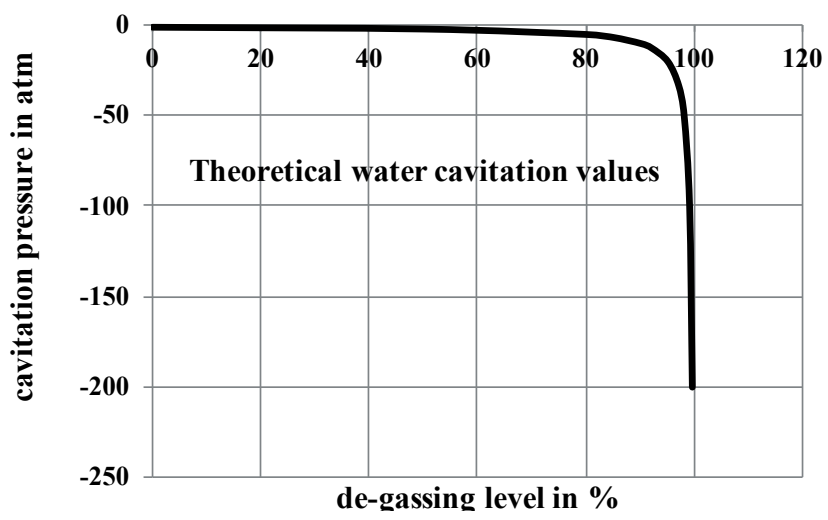

Figure 5. Effect of de-gassing on cavitation in water. densed liquids, in which the repulsive ideal-gas pressure $(P=n R T / V)$ is more than balanced by the cohesive molecular forces. For water at $20^{\circ} \mathrm{C}$, the 'ideal' repulsive molecular pressure $P$ is 1353 atm. Cohesive pressures in liquid water must be higher than this. Thus, there is strong evidence for this high value for pure, completely degassed water.

This analysis strongly suggests that the Galloway data $(1954)^{17}$, shown in Figure 3, is correct at relatively low degassing levels (less than 99\%) but that the cavitation pressures expected, for pure liquids, at higher levels of degassing was most likely not achieved in these experiments, probably due to the ubiquitous presence of contamination particles, which offer nucleation sites for cavity formation. Thus, the reported maximum of about $-200 \mathrm{~atm}$ is much less than the theoretical prediction.

3.2. Experimental study of the prevention of propeller cavitation in degassed water

The cavitation created by a three-blade propeller with a maximum speed of $2960 \mathrm{rpm}$ can be observed inside a water-filled perspex observation cell (Figure 6 and Figure 7). Tap water was pumped at $600 \mathrm{~mL} / \mathrm{min}$ through the hollow fibre membrane whilst vacuum was applied to its outer housing. The M700 oxygen analysis system displayed the measured oxygen content in the water tank, which continuously decreased.

When seawater was subjected to compressive pressures in the 10-55 atm range, we found in earlier studies $^{18}$ that on release of this pressure through a needle valve, cavitation of dissolved gases occurred. It was found that degassing the seawater to above $99 \%$ completely prevented this cavitation and this was used ${ }^{20}$ to improve the desalinated water product flow rate through a high pressure reverse osmosis system.

In these studies, it was found that by increasing the degassed level of the water, the speed of the propeller could be increased before cavitation was observed, as illustrated in Figure 8. The results of these rotation ate/degassing measurements are summarised in Table 1, which clearly shows that higher degassing levels produced higher cavitation pressures in the system (this data was obtained from the theoretical values for water cavitation in Figure 4). The experiments summarised in Table 1 were reproduced using a $0.5 \mathrm{M} \mathrm{NaCl}$ solution to simulate seawater. As an example, 70\% degassing was found to be sufficient to prevent cavitation even at the maximum rotation rate of $2960 \mathrm{rpm}$. This degree of degassing corresponds to a cavitation pressure of about 3 atm, according to Eq. (8). 


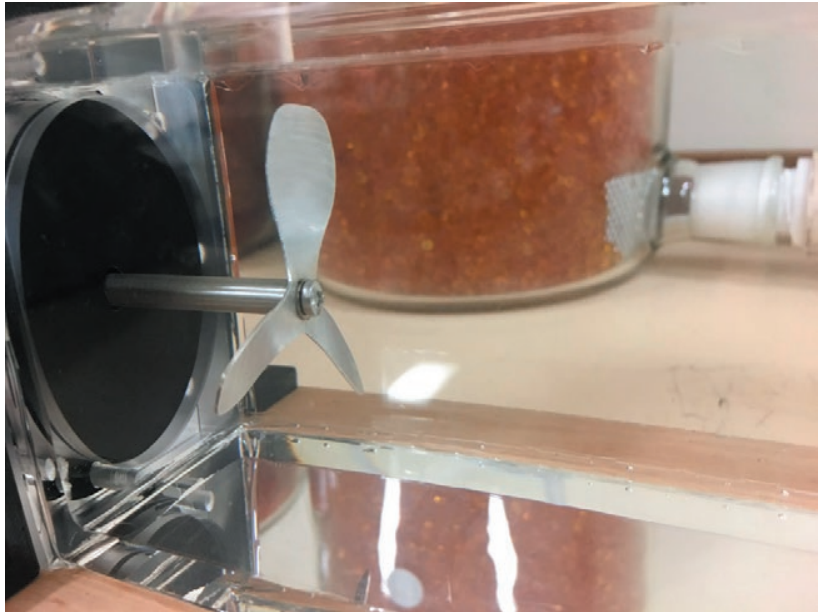

Figure 6. Photograph of the propeller used to study cavitation at the laboratory scale.

\subsection{Prevention of cavitation using a local degassed aque- ous environment}

In these experiments, degassed water was introduced locally, close to the low pressure side of rotating propeller blades immersed in gas-equilibrated water. Inside the perspex observation cell, gassed tap water flowed at a rate of $8 \mathrm{~L} / \mathrm{min}$ through a bent metallic pipe (10mm diameter), and $80 \%$ degassed water was released through a vertical metallic pipe with suitable holes (4.4 $\mathrm{mm}$ diameter and with six holes on the side and one at the end of the pipe) at a flow rate of about 1/10th the background water flow-rate, as shown in Figure 9. With only gassed tap water flowing within the cell, visible cavitation occurred (Figure 9), but when a local flow of degassed water was released adjacent to the propeller blades, all cavitation ceased (Figure 10). These experi-

Table 1. Experimental results of the de-gassing effects on cavitation observed for a rotating propeller completely immersed in de-gassed water.

\begin{tabular}{cccc}
\hline $\begin{array}{c}\text { Time } \\
(\mathrm{min})\end{array}$ & $\begin{array}{c}\text { De-gassing } \\
(\%)\end{array}$ & $\begin{array}{c}\text { Cavitation } \\
\text { pressure }(\mathrm{atm})\end{array}$ & $\begin{array}{c}\text { Minimum rpm to start } \\
\text { cavitation }\end{array}$ \\
\hline 0 & 0 & 1 & - \\
5 & 19.7 & 1.3 & 148 \\
10 & 27.8 & 1.5 & 592 \\
15 & 36.9 & 1.6 & 1628 \\
20 & 45.4 & 1.8 & 2072 \\
30 & 56.0 & 2.8 & 2500 \\
60 & 76.7 & 4 & Not even with 2960 \\
90 & 84.4 & 7 & Not even with 2960 \\
120 & 87.0 & 8 & Not even with 2960 \\
\hline
\end{tabular}

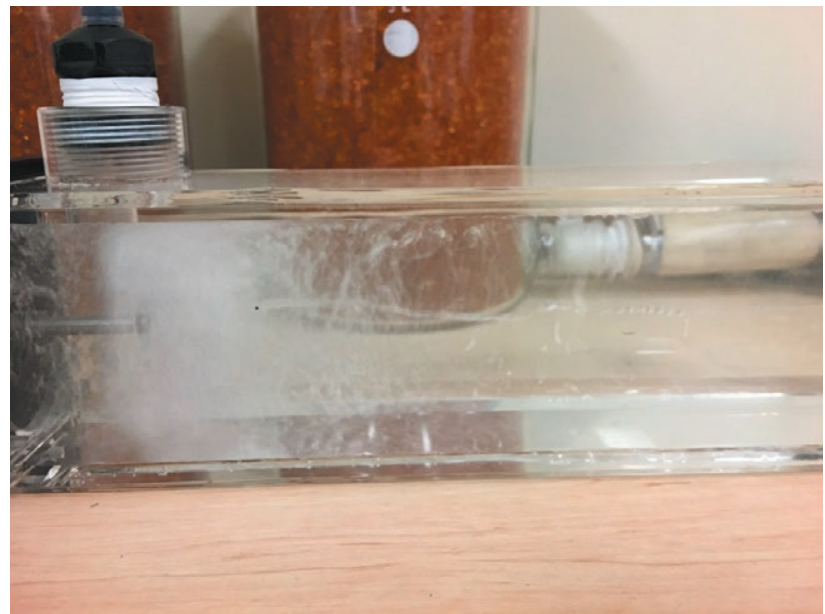

Figure 7. Cavitation occurring in air-equilibrated water at atmospheric pressure at a propeller rotation rate of $2960 \mathrm{rpm}$.

ments were carried out at high rotation rates of 2300 rpm using a three-blade propeller. The cessation of the sound accompanying cavitation on starting the degassed water flow was also obvious and immediate.

Acoustic measurements also show that the noise level was reduced from $75 \mathrm{~dB}$ to $65 \mathrm{~dB}$ after releasing the degassed water behind the propeller, despite having another, additional, noise involved which was from the water pump releasing the degassed water. The observed drop in $\mathrm{dB}$ level, even with the additional pump noise, corresponds to a sound intensity decrease on releasing the degassed fluid, of 10x. Similar results were also obtained using a $0.5 \mathrm{M} \mathrm{NaCl}$ aqueous solution to simulate seawater.

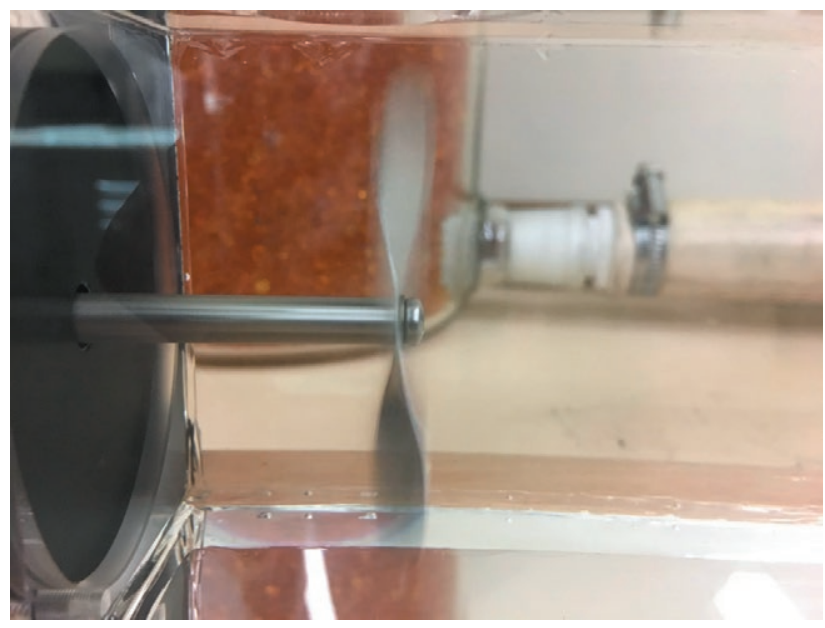

Figure 8. Complete cavitation prevention after gassed water was replaced with $70 \%$ de-gassed tap water (at $2960 \mathrm{rpm}$ propeller). 


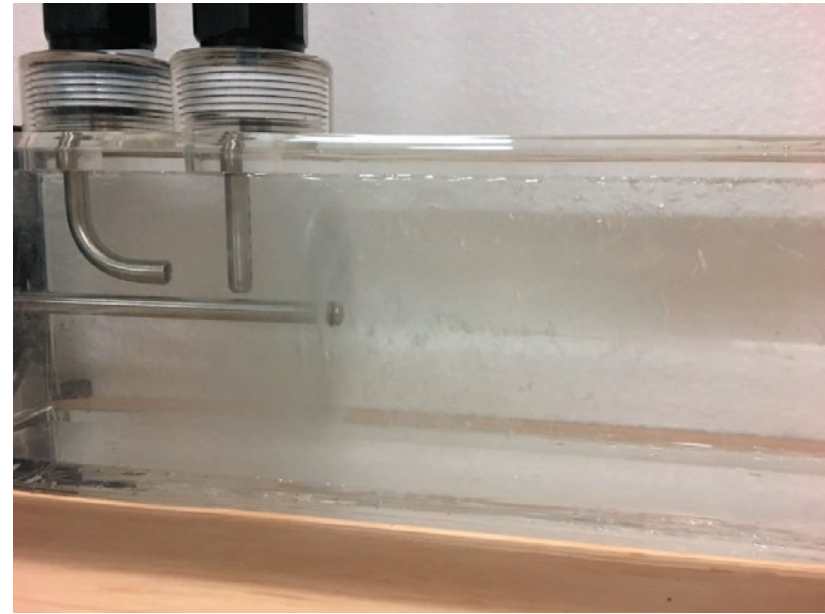

Figure 9. Cavitation occurring in flowing tap water at atmospheric pressure, i.e. in fully gassed water (at $2300 \mathrm{rpm}$ ).

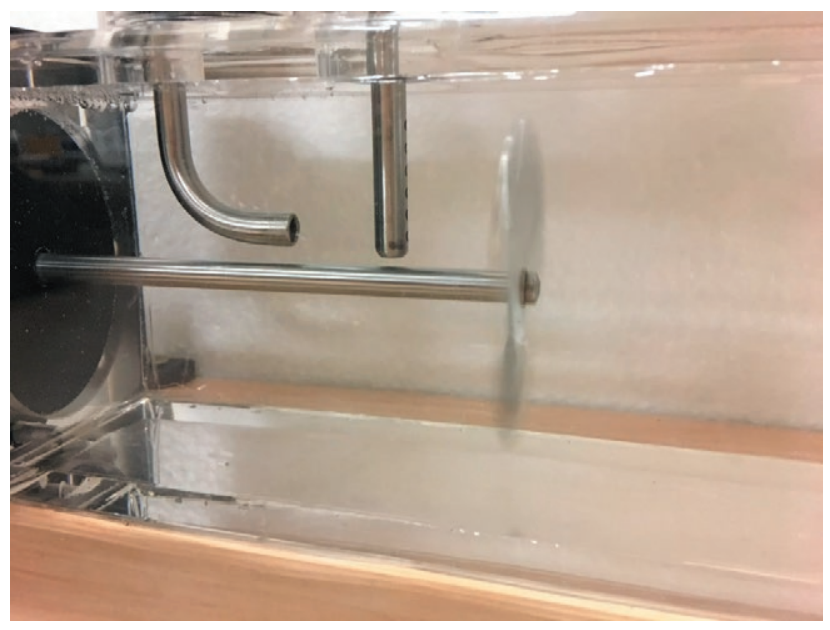

Figure 10. Cavitation completely prevented in gassed tap water after flowing $80 \%$ de-gassed water directly onto the rotating propeller blades (at $2300 \mathrm{rpm}$ ).

\subsection{Effects of degassed boundary layer films on a rotating} propeller blade

When the flow of degassed water was terminated, it was observed that the cavitation effects remained absent for several seconds or 100-200 rotations of the propeller. This is consistent with the observation (shown in Figure 10) that release of degassed water onto the upper rotating propeller blade prevented cavitation effects through a full cycle of the blade. These observations support the view that coating a rotating propeller with a film of degassed fluid is sufficient to give protection against cavitation.

The lowest pressure regions on the low pressure side of a rotating propeller blade are at the edge and the tip $u_{0}$

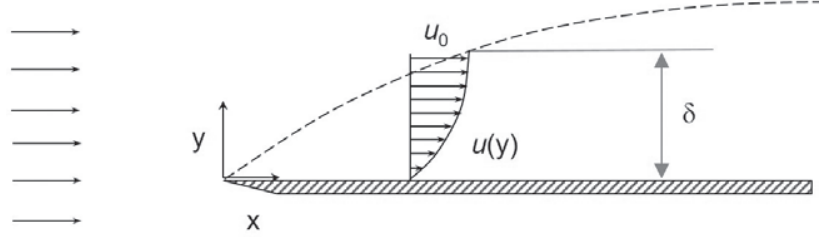

Figure 11. Schematic diagram of stationary or boundary-layer formation as a fluid flows over a flat solid surface (Adapted from David Weybourne (https://en.wikipedia.org/wiki/Boundary_layer_thick).

of the blade. For zero-slip conditions, water flow across a plate of length $x$ (i.e. the propeller blade), will slow down to zero at the surface, as shown in Figure 11.

The experimental boundary layer equation can be used to calculate the thickness $\delta$ of the water layer next to the plate at which the incident flow velocity reaches $99 \%$ of the bulk water flow velocity: $:^{19,20}$

$\delta=5 x / \sqrt{ }\left(R_{e}\right)$

where $R_{e}$ is the Reynolds number. This situation is shown in Figure 11, illustrating the 'zero-slip' condition, in which the fluid velocity slows to zero on the surface. The Reynolds number of the fluid depends on the distance $x$ the fluid has moved over the surface and these two parameters $\left(R_{e}, x\right)$ determine the thickness $\delta$ of the boundary layer. ${ }^{20}$

The Reynolds number in this case is given by

$R_{e}=\left(\rho V_{\infty} x\right) / \mu$

where $\rho$ is the density of water, $V_{\infty}$ the bulk fluid velocity (i.e. the incident fluid velocity beyond the boundary layer), $\mu$ is the dynamic viscosity of water and $x$ is the position on the surface.

Typically, $R_{e}$ corresponding to laminar flow will be in the range 1000 to $5 \times 10^{5}$. For example, if $R_{e}=50,000$, the boundary-layer water thickness $\mathrm{d}$ will be about 1.1 $\mathrm{cm}$ for an $x$ value of $0.5 \mathrm{~m}$. Much closer to the surface, say within $1 \%$ of the boundary layer thickness, the water moves only slowly relative to the solid surface: in a water layer of $110 \mu \mathrm{m}$ thickness, the water flow velocity is about $10 \mathrm{~cm} / \mathrm{s}$.

Cavitation occurs when dissolved gas is present in a fluid such as water. For the diffusion of gas in one direction $(x)$, the appropriate equation is Fick's second law in the form: ${ }^{21}$

$\frac{\partial C}{\partial t}=D \frac{\partial^{2} C}{\partial x^{2}}$ 
where $C$ is the solute concentration and $D$ the diffusion coefficient. When completely degassed water is exposed to air at atmospheric pressure, a very thin layer will form rapidly at the surface which will be at the atmospheric dissolved gas concentration (of $0.85 \mathrm{mM}$ ). This layer will maintain its saturated concentration from then onwards (in equilibrium with the atmosphere), and the dissolved gas will subsequently diffuse further into the water beyond this layer. Thus, Fick's law must be solved for the boundary conditions $C=C_{0}$, when $x=0$ for any value of $t$ and $C_{x}=0$ for $x>0$ when $t=0$. Also, $C_{x}=C_{0}$ for any value of $x$ as $t$ becomes very large. The solution to Fick's law under these conditions is: ${ }^{21}$

$C=C_{0}\left[1-\operatorname{erf}\left(\frac{x}{2 \sqrt{D t}}\right)\right]$

where the error function can be calculated using either tables or, as for the present calculation, using the series: ${ }^{21}$ $\operatorname{erf} x=\frac{2}{\sqrt{\pi}} \int_{0}^{x} e^{-t^{2}} d t=\frac{2 x}{\sqrt{\pi}}\left[1-\frac{x^{2}}{1 \times 3}+\frac{x^{4}}{2 \times 1 \times 5}-\right.$ $\left.\frac{x^{6}}{3 \times 2 \times 1 \times 7}+\cdots\right]$

For oxygen and nitrogen gases in water the value of the diffusion coefficient $D$ at $20^{\circ} \mathrm{C}$ is about $2 \times 10^{-5}$ $\mathrm{cm}^{2} / \mathrm{s}$ and the saturated gas concentration, $C_{0}$ is about $0.85 \mathrm{mM}$. Using these values, the calculated results obtained show that for quiescent water that is in the almost stationary part of the boundary layer, closest to the solid surface, significant re-gassing of a degassed water film of about $100 \mu \mathrm{m}$ thickness would take several seconds.

Thus, a transient coating of a boundary layer of degassed water on a rapidly rotating blade can be used to prevent cavitation effects for many subsequent rotations. This means that the amount of degassed water required to prevent or minimise cavitation can be substantially reduced, and the effect of cavitation minimisation can persist for a significant period of time e.g. a few or more seconds. The effect of reducing the occurrence of cavitation will persist until the stationary film drains as the blade rotates or until diffusion from the bulk fluid re-gasses the degassed film.

The effects of having a transient film of degassed fluid could be further optimised by having a periodic, controlled release of a positive, relatively high pressure flow of degassed fluid, released close to the rotating blades and timed to strike the leading edge on the negative pressure side. Once the de-gassed fluid strikes the blade, it would coat the face with degassed fluid (e.g. water) and form a boundary layer. The zeroslip boundary condition will ensure the retention of a degassed film on the rotating surface. Put another way, the degassed fluid could be periodically directed onto the surface using a pulsed flow to form the boundary layer. Use of a pulsed flow of degassed fluid may mean degassed fluid only needs to be released in a periodic fashion, such as every 1 in 100 rotations of the blade, or for only $1 \%$ of the time.

The leading edge of the rotating blade divides the incident flow into a high pressure stream on the nearly flat 'lower' face and a low pressure stream on the upper side of the blade. As the blade rotates, the film of degassed water will drain from the leading edge to the trailing edge of the blade, and suppress cavitation across the low pressure face. At the same time, dissolved gases within the flowing fluid (e.g. water) begin to diffuse into the degassed boundary layer. However, gas diffusion under effectively quiescent conditions next to the solid surface is relatively slow; for a $0.1 \mathrm{~mm}$ film, this re-gassing will take several seconds.

In addition to these effects, the centrifugal forces generated by the rotating blade will force the degassed boundary layer to flow towards the tip of the rotating blade, which will also help to prevent cavitation at this point, where it is often observed.

\section{CONCLUSIONS}

This study has demonstrated a new method for preventing cavitation at a surface that moves relative to a fluid. The method comprises directing a second fluid that is at least partially degassed towards the surface, where it will form a boundary layer. The boundary layer at least partially increases the negative pressure required to initiate cavitation at the surface, reducing the occurrence of cavitation during the relative movement. Collapse of the cavities formed during cavitation creates shockwaves, and hence microjets, which impinge upon surfaces, creating noise and causing wear, for example, on propeller blades. In these experiments, noise generation was completely suppressed, which suggests that wear could also be substantially reduced. Therefore, this process may help to reduce cavitation noise and wear and improve the efficiency of propeller-driven boats, ships and submarines.

\section{ACKNOWLEDGEMENTS}

The authors would like to acknowledge Mechanical Workshop staff at the School of Science at UNSW Canberra. 


\section{REFERENCES}

1. H. Kato, Cavitation, in Advances in Marine hydrodynamics, Computational mechanics publications, 1996.

2. C.E. Brennen, Cavitation and Bubble Dynamics, Oxford University Press, 1995.

3. L. Rayleigh, VIII. On the pressure developed in a liquid during the collapse of a spherical cavity, The London, Edinburgh, and Dublin Philosophical Magazine and Journal of Science, 1917, 34 (200), 94-98.

4. R. M. Pashley, P. M. McGuiggan, B. W. Ninham, D. F. Evans, Attractive forces between uncharged hydrophobic surfaces: direct measurements in aqueous solution, Sci., 1985, 229(4718), 1088-1089.

5. R. W. Johnson, Handbook of Fluid Dynamics, 1998, CRC Press.

6. Z. Pan, A. Kiyama, Y. Tagawa, D. J. Daily, S. L. Thomson, R. Hurd, T. T. Truscott, Cavitation onset caused by acceleration, Proc. Nat. Acad. Sci., 2017, 114(32), 8470-8474.

7. J. N. Israelachvili, R. M. Pashley, The hydrophobic interaction is long range, decaying exponentially with distance, Nature, 1982, 300(5890), 341-342.

8. H. K. Christenson, J. N. lsraelachvili, R. M. Pashley, Properties of Capillary Fluids at the Microscopic Level, SPE Res. Eng., 1987, 155-165.

9. M. Alheshibri, J. Qian, M. Jehannin, V.S.J. Craig, A history of nanobubbles, Langmuir, 2016, 32, 11086-11100.

10. A. Agarwal, W. J. Ng, Y. Liu, Principle and applications of microbubble and nanobubble technology for water treatment, Chemosphere, 2011, 84, 1175-1180.

11. Z. Fang, X. Wang, L. Zhou, L. Zhang, J. Hu, Formation and Stability of Bulk Nanobubbles by Vibration, Langmuir, 2020, 36, 2264-2270.

12. R. M. Pashley, Effect of degassing on the formation and stability of surfactant-free emulsions and fine teflon dispersions, J. Phys. Chem. B, 2003, 107(7), 17141720.

13. J. Antony, R. M. Pashley, M. Taseidifar, Prevention of Cavitation, World Intellectual Property Organization, 2018, WO2018191790

14. M. Rzechowicz, R. M. Pashley, A membrane method for degassing nonaqueous liquids, J. Colloid Interface Sci., 2006, 298(1), 321-326.

15. M. Rzechowicz, R. M. Pashley, The effect of de-gassing on the efficiency of reverse osmosis filtration, $J$. Membr. Sci., 2007, 295(1), 102-107.

16. Q. Zheng, D.J. Durben, G.H. Wolf, C.A. Angell, Liquids at Large Negative Pressures: Water at the Homogeneous Nucleation Limit, Sci., 1991, 254(5033), 829832.
17. W.J. Galloway, An Experimental Study of Acoustically Induced Cavitation in Liquids, J. Acoust. Soc. Am., 1954, 26(5) 849-857.

18. M.J. Francis, R.M. Pashley, M. Rzechowicz, The effects of feed water de-gassing on the permeate flux of a small scale SWRO pilot plant. Desalination and Water Treat., 2011, 25(1-3) 150-158.

19. J.H. Arakeri, P.N. Shankar, Ludwig Prandtl and boundary layers in fluid flow. Resonance, 2000, 5(12) 48-63.

20. H. Schlichting, K. Gersten, Fundamentals of Boundary-Layer Theory, in Boundary-Layer Theory, Springer Berlin Heidelberg: Berlin, Heidelberg, 2017, 29-49.

21. R. M. Pashley, M. Rzechowicz, L. R. Pashley, M. J. Francis, De-Gassed Water Is a Better Cleaning Agent, J. Phys. Chem. B, 2005, 109(3) 1231-1238. 